\title{
STABILITY THEORY FOR FUNCTIONAL DIFFERENTIAL EQUATIONS
}

\author{
BY
}

T. A. BURTON

\begin{abstract}
We consider a system of functional differential equations $x^{\prime}(t)=$ $\mathscr{F}(t, x(\cdot))$, together with a Liapunov functional $\mathfrak{T}(t, x(\cdot))$ with $\mathcal{V}^{\prime}<0$. Most classical results require that $\mathcal{F}$ be bounded for $x(\cdot)$ bounded and that $\mathscr{F}$ depend on $x(s)$ only for $t-\alpha(t)<s<t$ where $\alpha$ is a bounded function in order to obtain stability properties. We show that if there is a function $H(t, x)$ whose derivative along $x^{\prime}(t)=\mathscr{F}(t, x(\cdot))$ is bounded above, then those requirements can be eliminated. The derivative of $\boldsymbol{H}$ may take both positive and negative values. This extends the classical theorem on uniform asymptotic stability, gives new results on asymptotic stability for unbounded delays and unbounded $\mathcal{F}$, and it improves the standard results on the location of limit sets for ordinary differential equations.
\end{abstract}

1. Introduction. In this section we describe our results and their relation to results of previous investigators. Definitions and background may be found in the next section. Throughout the paper, functionals will be denoted by script letters and functions by italic and Greek letters.

We consider a system of functional differential equations

$$
x^{\prime}(t)=\mathscr{F}(t, x(s) ; \alpha \leqslant s \leqslant t), \quad 0 \leqslant t<\infty,
$$

where $\mathscr{F}$ is a continuous functional defined and taking values in $R^{n}$ whenever $t \in[0, \infty)$ and $x$ is a continuous function with $x:[\alpha, \infty) \rightarrow R^{n}$. Our notation follows that of Driver [4, pp. 403-426]. If $\alpha=-\infty$, then it is understood that $x:(-\infty, t] \rightarrow R^{n}$.

The results concern behavior of solutions of (1) when there is a Liapunov functional $\mathcal{V}(t, x(t))$ whose derivative along solutions of $(1)$ is nonpositive. Most of the classical results on asymptotic stability require that $\mathscr{F}(t, x(\cdot))$ be bounded for $x$ bounded and that $\mathscr{F}$ depend on $x(s)$ only for $t-\alpha(t) \leqslant s \leqslant t$ with $\alpha(t)$ bounded.

In this paper we show that if there is a function (not a functional) $H(t, x)$ which is only mildly dependent on $t$ with $H_{(1)}^{\prime}(t, x)$ bounded above for $x(\cdot)$ bounded, then the bounds on $\mathcal{F}$ and $\alpha(t)$ can be eliminated. As we allow $H^{\prime}$

Received by the editors December 15, 1977 and, in revised form, September 11, 1978.

AMS (MOS) subject classifications (1970). Primary 34K20, 34D20.

Key words and phrases. Stability, Liapunov functions, ordinary differential equations, delay equations.

(c) 1979 American Mathematical Society 0002-9947/79/0000-0511/\$04.25 
to be both positive and negative, we do not ask for two Liapunov functions in the sense that previous authors have.

Theorem 1 yields the classical asymptotic stability result of Marachkov for ordinary differential equations as a corollary.

Theorem 2 deals with (1) in its general form; however, when restricted to an ODE it generalizes that class of results typified by Theorem 1 of La Salle $[10$, p. 60], and Theorem 14.1 of Yoshizawa [12, p. 60] (or Theorem 5 of [13, p. 382]) which we paraphrase as follows:

THEOREM 0 . Let $V(t, x)$ be a differentiable scalar function which is nonnegative and which satisfies $V^{\prime}(t, x) \leqslant-W(x) \leqslant 0$ along solutions of $x^{\prime}=P(t, x)$. If $|P(t, x)|$ is bounded for $|x|$ bounded, then each bounded solution approaches $E=\{x \mid W(x)=0\}$.

Theorem 3 is a generalization of the classical result of Yoshizawa on uniform asymptotic stability of a system of functional differential equations $x^{\prime}(t)=\mathcal{G}\left(t, x_{t}\right)$.

In order to conserve space and simplify notation, Theorems 1 and 2 are stated in terms of the whole space. There is no difficulty in restricting the hypotheses to a subset $G$ and concluding that solutions remaining in $G$ have the specified properties. Likewise, we ask $\mathcal{V} \geqslant 0$; however, one may ask $\mathfrak{V}$ bounded from below for $x(\cdot)$ bounded. The results and hypotheses are identical for bounded solutions.

2. Definitions, assumptions, and setting. Following the convention and notation of Driver [4, p. 403], a functional

$$
\text { थ }(t, x(s) ; \alpha \leqslant s \leqslant t), \quad 0 \leqslant t<\infty,
$$

will be called:

(a) continuous in $t$ and $x$ if $\mathscr{U}(t, x(\cdot))$ is a continuous function of $t$ for $0 \leqslant t<\infty$ whenever $x:[\alpha, \infty) \rightarrow R^{n}$ is continuous,

(b) locally Lipschitz in $x$ if for every $T>0$ and every compact set $D \subset R^{n}$ there exists a constant $L_{T, D}$ with

$$
|थ(t, x(\cdot))-\mathscr{Q}(t, y(\cdot))| \leqslant L_{T, D}\|x-y\|^{[\alpha, t]}
$$

whenever $t \in[0, T]$ and $x, y:[\alpha, T] \rightarrow D$.

Here, if $u \in R^{n}$, then $|u|=\max _{i}\left|u_{i}\right|$ and if $\psi:[a, b] \rightarrow R^{n}$, then

$$
\|\psi\|^{[a, b]}=\sup _{a<s<b}|\psi(s)| \text {. }
$$

When $\mathcal{F}$ is continuous and locally Lipschitz, if $t_{0} \geqslant 0$ and if $\phi:\left[\alpha, t_{0}\right] \rightarrow R^{n}$ is continuous, then there is a solution $x\left(t, t_{0}, \phi\right)$ of (1) on an interval $\left[t_{0}, \beta\right)$ with $x\left(t, t_{0}, \phi\right)=\phi(t)$ for $\alpha \leqslant t \leqslant t_{0}$. ( $\phi$ is called the initial function.) This solution can be continued to an interval $[\alpha, T)$ with $T=\infty$ or 
(cf. Driver [4, p. 408]).

$$
\varlimsup_{t \rightarrow T^{-}}\left|x\left(t, t_{0}, \phi\right)\right|=\infty
$$

Assumption A. Throughout this paper we assume $\mathscr{F}$ smooth enough that a solution exists for each continuous initial condition and that if it remains bounded it can be continued to $\infty$.

Equation (1) includes delay equations

$$
x^{\prime}(t)=F(t, x(t), x(t-r(t)))
$$

with $0 \leqslant r(t) \leqslant t$ and $r(t)$ continuous; it includes functional differential equations

$$
x^{\prime}(t)=\mathcal{G}\left(t, x_{t}\right)
$$

where $x_{t}$ is the segment of $x(s)$ for $t-h \leqslant s \leqslant t$ shifted back to [ $\left.-h, 0\right]$ as developed by Yoshizawa [12, pp. 183-213]; and it includes ordinary differential equations

$$
x^{\prime}(t)=P(t, x(t)) .
$$

Assumption A holds for (2) and (4) if $F$ and $P$ are continuous. It holds for (3) if $\mathcal{G}$ takes bounded sets into bounded sets and $\mathcal{G}$ is continuous.

Our work is based on the assumption that there exists a continuous scalar functional

$$
\mathcal{V}(t, x(s) ; \alpha \leqslant s \leqslant t), \quad 0 \leqslant t<\infty,
$$

which is defined and continuous for $0 \leqslant t<\infty$ whenever $x$ is a continuous function with $x:[\alpha, t) \rightarrow R^{n}$ and which is locally Lipschitz in $x$.

The derivative of $\mathfrak{V}$ along a solution $\psi(t)$ of (1) is defined by Driver [4, p. 414] as

$$
\mathcal{V}_{(1)}^{\prime}(t, \psi(\cdot))=\varlimsup_{\Delta t \rightarrow 0} \frac{\mathscr{V}\left(t+\Delta t, \psi^{*}(\cdot)\right)-\mathscr{V}(t, \psi(\cdot))}{\Delta t}
$$

where

$$
\psi^{*}(s)= \begin{cases}\psi(s) & \text { for } \alpha<s<t, \\ \psi(t)+\mathscr{F}(t, \psi(\cdot))(s-t) & \text { for } t<s \leqslant t+\Delta t\end{cases}
$$

For more details regarding existence, uniqueness, and implications of this limit see Driver [4, pp. 414-415], Hale [7, p. 105], and Yoshizawa [12, pp. 186-187]. In many concrete problems $\mathcal{V}_{(1)}^{\prime}$ is a simple application of the chain rule.

We suppose that

$$
\mathscr{V}_{(1)}^{\prime}(t, x(\cdot)) \leqslant 0 \text { and } \mathscr{V}(t, x(\cdot))>0 .
$$

If $\mathfrak{V}$ satisfies these conditions it is called a Liapunov functional.

Summaries of results on existence and stability are found in [4], [5], [7, pp. 105-140], and [12, pp. 183-213]. The reader is especially referred to [4, p. 415, 
Theorem 4] for one of the few existing asymptotic stability results for unbounded delays.

When $\mathscr{F}(t, 0) \equiv 0$, then $x(t) \equiv 0$ is a solution of (1). The following terms are then used with some frequency.

The solution $x(t) \equiv 0$ of (1) is:

(a) stable if for each $t_{0}>0$ and each $\varepsilon>0$ there exists $\delta=\delta\left(\varepsilon, t_{0}\right)>0$ such that if $\phi$ is a continuous initial function with $\|\phi\|^{\left[\alpha, t_{0}\right]}<\delta$, then $\left|x\left(t, t_{0}, \phi\right)\right|<\varepsilon$ for $t \geqslant t_{0}$,

(b) uniformly stable if $\delta=\delta(\varepsilon)$,

(c) asymptotically stable if it is stable and if there exists $\delta_{1}=\delta_{1}\left(t_{0}\right)>0$ such that if $\|\phi\|^{\left[\alpha, t_{0}\right]}<\delta_{1}$, then $x\left(t, t_{0}, \phi\right) \rightarrow 0$ as $t \rightarrow \infty$.

(d) uniformly asymptotically stable (UAS) if it is uniformly stable, if $\delta_{1}$ is independent of $t_{0}$, and if for every $\eta>0$ there exists $T(\eta)>0$ such that for $t_{0} \geqslant 0$ and $\|\phi\|^{\left[\alpha, t_{0}\right]}<\delta_{1}$, then $\left|x\left(t, t_{0}, \phi\right)\right|<\eta$ when $t \geqslant t_{0}+T(\eta)$.

Throughout the remainder of the paper we use continuous functions, called wedges,

$$
W_{i}:[0, \infty) \rightarrow[0, \infty)
$$

with $W_{i}(0)=0, W_{i}(s)>0$ if $s>0$, and $W_{i}$ strictly increasing.

A function $U:[0, \infty) \times G \rightarrow[0, \infty)$, with $G \subset R^{n}$, is called

(a) positive definite if $U(t, 0) \equiv 0$ and if there is a wedge $W_{1}$ with $U(t, x) \geqslant$ $W_{1}(|x|)$.

(b) decrescent if there is a wedge $W_{2}$ with $U(t, x) \leqslant W_{2}(|x|)$.

(c) negative definite if $-U(t, x)$ is positive definite.

The literature is not uniform concerning definite properties of functionals, particularly when the delay is unbounded. The conflict mainly concerns the requirement on $\mathcal{V}_{(1)}^{\prime}(t, x(\cdot))$. Driver defines $\mathcal{V}_{(1)}^{\prime}(t, x(\cdot))$ to be negative definite if there is a wedge $W_{3}$ with $\mathcal{V}_{(1)}^{\prime}(t, x(\cdot)) \leqslant-W_{3}(|x(t)|)$. Such a condition is frequently satisfied in examples, but then much additional work and additional assumptions are normally needed to conclude stability properties. Other authors ask a variant of $\mathcal{V}_{(1)}^{\prime}(t, x(\cdot)) \leqslant-W_{4}\left(\|x\|^{[t-h, t]}\right)$ for some $h>0$. Such a requirement is often satisfied in converse theorems, and it does easily imply stability properties. However, it is seldom realized in concrete problems.

3. Limit sets and stability. In our first result we suppose that there is a differentiable function

$$
H: R^{n} \rightarrow(-\infty, \infty)
$$

and a continuous function $K:[0, \infty) \rightarrow[0, \infty)$ with

$$
\operatorname{grad} H(x(t)) \cdot \mathcal{F}(t, x(s) ; \alpha \leqslant s \leqslant t) \leqslant K(M)
$$

for $\alpha \leq s \leqslant t$ whenever $x$ is a continuous function satisfying $|x(s)| \leqslant M$. 
THEOREM 1. Let $\mathcal{V}$ be a functional satisfying (6) with $\mathcal{V}_{(1)}^{\prime}(t, x)<$ $-W_{4}(|x(t)|)$ and suppose there is a function $H$ satisfying (8) and (9). Then each solution $x(t)$ of (1) bounded on $\left[t_{0}, \infty\right)$ satisfies $H(x(t)) \rightarrow H(0)$ as $t \rightarrow \infty$.

Proof. Notice first that the new function $H(x)-H(0)$ also satisfies (8) and (9). Thus, we suppose $H(0)=0$. If the theorem is false, then there is a solution $x(t)$ of (1) on $\left[t_{0}, \infty\right)$ with $|x(t)| \leqslant M$ for some $M>0$, an $\varepsilon>0$, and a sequence $\left\{T_{n}\right\}$ tending to infinity with $\left|H\left(x\left(T_{n}\right)\right)-H(0)\right| \geqslant \varepsilon$. As we have supposed $H(0)=0$, we have $\left|H\left(x\left(T_{n}\right)\right)\right| \geqslant \varepsilon$. Suppose first that there is a subsequence, say $\left\{T_{n}\right\}$ again, with $H\left(x\left(T_{n}\right)\right) \geqslant \varepsilon$.

Now $\mathcal{V} \geqslant 0$ and $\mathcal{V}_{(1)}^{\prime}(t, x) \leqslant-W_{4}(|x(t)|)$ so

$$
0 \leqslant \mathcal{V}(t, x) \leqslant \mathcal{V}\left(t_{0}, \phi\right)-\int_{t_{0}}^{t} W_{4}(|x(s)|) d s .
$$

Thus, $x(t)$ is not bounded strictly away from zero. There is a sequence $\left\{t_{n}\right\}$ tending to infinity such that $x\left(t_{n}\right) \rightarrow 0$. Hence, $H\left(x\left(t_{n}\right)\right) \rightarrow 0$. As $H$ is continuous, there exists $\delta>0$ with $|H(x)|<\varepsilon / 2$ if $|x|<\delta$.

We can find sequences $\left\{s_{n}\right\}$ and $\left\{S_{n}\right\}$ increasing to infinity with $H\left(x\left(s_{n}\right)\right)$ $=\varepsilon / 2, H\left(x\left(S_{n}\right)\right)=3 \varepsilon / 4$, and $|x(t)| \geqslant \delta$ if $s_{n} \leqslant t \leqslant S_{n}$. That is, $H(x(t))$ is continuous and, if $t_{k}<T_{j}$, then $H(x(t))$ moves from near zero to near $\varepsilon$ as $t$ goes from $t_{k}$ to $T_{j}$ when $k$ is large.

As $H_{(1)}^{\prime}(x) \leqslant K(M)$ on each interval $\left[s_{n}, S_{n}\right]$ (as seen from (9) and the chain rule), we obtain

$$
\varepsilon / 4=H\left(x\left(S_{n}\right)\right)-H\left(x\left(s_{n}\right)\right) \leqslant K(M)\left(S_{n}-s_{n}\right)
$$

or

$$
S_{n}-s_{n} \geqslant \varepsilon / 4 K(M) \stackrel{\text { def }}{=} T .
$$

If $H\left(x\left(T_{n}\right)\right) \leqslant-\varepsilon$, then choose $\left\{S_{n}\right\}$ and $\left\{s_{n}\right\}$ with $H\left(x\left(s_{n}\right)\right)=-3 \varepsilon / 4$, $H\left(x\left(S_{n}\right)\right)=-\varepsilon / 2$, and $s_{n}<S_{n}$. Proceed as before to $S_{n}-s_{n} \geqslant T$.

As $\mathcal{V}_{(1)}^{\prime}(t, x) \leqslant-W_{4}(|x(t)|) \leqslant-W_{4}(\delta)$ for $s_{n} \leqslant t \leqslant S_{n}$, if we integrate from $t_{0}$ to $t>S_{n}$ we obtain

$$
\begin{aligned}
\mathcal{V}(t, x) & \leqslant \mathcal{V}\left(t_{0}, \phi\right)-\int_{t_{0}}^{t} W_{4}(|x(s)|) d s \\
& \leqslant \mathcal{V}\left(t_{0}, \phi\right)-\sum_{i=1}^{n} \int_{s_{i}}^{s_{i}} W_{1}(\delta) d s \leqslant \mathscr{V}\left(t_{0}, \phi\right)-n T W_{4}(\delta)
\end{aligned}
$$

which tends to $-\infty$ as $n \rightarrow \infty$. This contradiction completes the proof.

EXAMPLE 0. In the scalar delay equation

$$
x^{\prime}(t)=-\left(t^{2}+4\right) x(t)+x(t-t / 2)
$$


we take

$$
\mathcal{V}(t, x)=x^{2}(t)+\int_{t / 2}^{t} x^{2}(s) d s
$$

and obtain

$$
\begin{aligned}
\mathcal{V}^{\prime}(t, x) & =-2\left(t^{2}+4\right) x^{2}(t)+2 x(t) x(t / 2)+x^{2}(t)-\frac{1}{2} x^{2}(t / 2) \\
& \leqslant-5 x^{2}(t) \stackrel{\text { def }}{=}-W_{4}(|x(t)|) .
\end{aligned}
$$

The last inequality was obtained by completing the square. Then take $H(x)=x^{2}$ and obtain $H^{\prime} \leqslant+2 x(t) x(t / 2)$ which is bounded above for $x(\cdot)$ bounded. As $\mathcal{V} \geqslant x^{2}$ and $\mathcal{V}^{\prime} \leqslant 0$, all solutions are bounded. Thus, all tend to zero.

The example is elementary and the conclusion is certainly expected; yet, known results on Liapunov functionals do not seem to yield it because of $t^{2}+4$ and $t-t / 2$ being unbounded. In any case, it is a simple illustration of the theorem.

EXAmple 1. Consider the differential equation (4) and suppose that $J$ is a Liapunov function for (4):

$$
\begin{gathered}
J:[0, \infty) \times R^{n} \rightarrow[0, \infty), \quad J_{(4)}^{\prime}(t, x)<-W_{4}(|x(t)|), \\
J(t, 0)=0, \quad J(t, x)>W_{1}(|x|) .
\end{gathered}
$$

Let $P(t, x)=\left(P_{1}, \ldots, P_{n}\right)$ and suppose that some $P_{i}$ is bounded for $x$ bounded. Now $x=0$ is Liapunov stable. Let $x(t)$ be a bounded solution of (4) and suppose $x(t)=\left(x_{1}, \ldots, x_{n}\right)$. Then $x_{i}(t) \rightarrow 0$.

To see this, take $H(x)=x_{i}^{2}$ so that $H_{(4)}^{\prime}(x)=2 x_{i} P_{i}(t, x)$ which is bounded for $x$ bounded. Hence, $x_{i}^{2}(t) \rightarrow 0$ as $t \rightarrow \infty$.

Corollary (MarachKov \{CF.[1,TheORem 7, p. 149]\}). If $J(t, x)$ is positive definite, if $J_{(4)}^{\prime}(t, x)$ is negative definite, and if $P(t, x)$ is bounded for $|x|$ bounded, then $x=0$ is asymptotically stable.

Proof. Take $H(x)=x_{1}^{2}+\cdots+x_{n}^{2}$ and obtain $H_{(4)}^{\prime}(x)$ bounded for $x$ bounded. Thus, $H(x(t)) \rightarrow H(0)=0$ and so bounded solutions approach zero. As $J$ is positive definite and $J_{(4)}^{\prime} \leqslant 0, x=0$ is Liapunov stable. This completes the proof.

The effect of this corollary is that if the conditions of Marachkov's result hold, then the conditions of Theorem 1 hold. The reverse is, of course, false.

Marachkov's result yielded asymptotic stability when $J$ was not decrescent. Results of the class of Theorem 0 were direct extensions of Marachkov's result. These extensions introduced the set $E$ in place of $\{0\}$ as the location where $J^{\prime}$ could tend to zero. They also replaced $J$ positive definite by the (tacit) assumption that some solutions were bounded. Except in cases where 
$J^{\prime}$ was related to $|P|$ (cf. Haddock [6]), these extensions demanded $P$ bounded for $x(t)$ bounded. (A recent paper by La Salle [11, p. 84] gives a condition requiring the integral of $P$ bounded over certain functions to effect the same conclusion.)

Our result is an extension of Marachkov's in an entirely different direction. It replaces $\mathscr{F}$ (or $P$ ) bounded with the requirement (8) and (9); the conclusion still involves zero instead of a general set $E$. Our next result generalizes Marachkov's result by again allowing $\mathcal{F}$ (or $P$ ) to be unbounded, but also allowing $E$ to be a general closed set in $R^{n}$. However, we now need restrictions on $\boldsymbol{H}$.

We pattern our formulation after that given by Yoshizawa [12, pp. 116-167] for stability of a compact set $E$.

In the following, $N(a, E)$ denotes the $a$-neighborhood of a set $E, N^{c}(a, E)$ denotes its complement, and $d(x, E)$ is the distance from $x$ to $E$.

Definition. Let $E \subset R^{n}$ be a closed set, $U$ an open neighborhood of $E$, and let $H:[0, \infty) \times U \rightarrow[0, \infty)$ be a differentiable function. $H$ is a pseudoLiapunov function for (1) and $E$ if for each compact subset $K$ of $U$ :

(i) for any $\varepsilon>0$, there exists $\lambda>0$ such that $H(t, x)>\lambda$ for $x \in K \cap$ $N^{c}(\varepsilon, E)$,

(ii) for any $\lambda>0$, there exists $\eta>0$ such that $H(t, x)<\lambda / 2$ for $x \in K \cap$ $N(\eta, E)$, and

(iii) if $x(t)$ is a bounded solution of (1) on $\left[t_{0}, \infty\right)$ then for $x(t) \in K$ we have $\operatorname{grad} H \cdot \mathcal{F}+\partial H / \partial t$ bounded above.

If $H$ is only locally Lipschitz, then in (iii) we ask $H_{(1)}^{\prime}$ bounded above instead of $\operatorname{grad} H \cdot F+\partial H / \partial t$ bounded above.

EXAMPLE 2. In the system

$$
x_{1}^{\prime}=x_{2}, \quad x_{2}^{\prime}=-\psi\left(t, x_{1}(t), x_{2}(t)\right) x_{2}(t)-g\left(x_{1}(t-r(t))\right),
$$

let $\psi \geqslant 0, \psi, g$, and $r$ be continuous, and $t \geqslant r(t) \geqslant 0$. If $E=\left\{\left(x_{1}, x_{2}\right) \mid x_{2}=\right.$ $0\}$ and $H\left(t, x_{1}, x_{2}\right)=x_{2}^{2}$, then (i) and (ii) hold, while

$$
\begin{aligned}
H_{(10)}^{\prime} & =-2 \psi(\cdot) x_{2}^{2}-2 x_{2} g\left(x_{1}(t-r(t))\right) \\
& \leqslant-2 x_{2} g\left(x_{1}(t-r(t))\right)
\end{aligned}
$$

so that if $x(t)$ is bounded on $\left[t_{0}, \infty\right)$, then $H^{\prime}$ is bounded above. Thus, $H$ is a pseudo-Liapunov function for (10).

Definition. A function $L: R^{n} \rightarrow[0, \infty)$ is positive definite relative to a closed set $E \subset R^{n}$ if for any compact set $K \subset R^{n}$ and any $\varepsilon>0$, there exists $L_{0}>0$ such that $L(x)>L_{0}$ for $x \in K \cap N^{c}(\varepsilon, E)$.

THEOREM 2. Let $\mathcal{V}$ be a functional satisfying (5) and (6) with $\mathcal{V}_{(1)}^{\prime}(t, x)<$ - $L(x(t))$ where $L$ is positive definite relative to a closed set $E \subset R^{n}$. If $U$ is an open neighborhood of $E$ and $H:[0, \infty) \times U \rightarrow[0, \infty)$ is a pseudo-Liapunov 
function for (1) and $E$, then each bounded solution of (1) approaches $E$ as $t \rightarrow \infty$.

Proof. If the theorem is false, then there is a bounded solution $x(t)$ of (1) which does not approach $E$. Then there is a compact set $\bar{K} \subset R^{n}$ with $x(t) \in \bar{K}$ for $t_{0} \leqslant t<\infty$. Also, there is an $\varepsilon>0$ with $N(2 \varepsilon, E) \cap \bar{K} \subset U$ and a sequence $\left\{\bar{t}_{n}\right\}$ tending to infinity with $d\left(x\left(\bar{t}_{n}\right), E\right)=\varepsilon$. (That is, we first say that $d\left(x\left(t_{n}\right), E\right) \geqslant \varepsilon$; but as $\mathcal{V}^{\prime} \leqslant-L(x(t))$ we argue that $x(t)$ approaches $E$ along a sequence. Thus, we can select $\bar{t}_{n}$ so that equality holds.) Let $K=\bar{K} \cap \bar{N}(\varepsilon, E)$ where $\bar{N}$ is the closure of $N$. For this compact subset $K$ of $U$ and this $\varepsilon>0$, find $\lambda$ and $\eta$ of parts (i) and (ii) of the definition of a pseudo-Liapunov function with $0<2 \eta<\varepsilon$.

As $\mathcal{V}_{(1)}^{\prime}(t, x) \leqslant-L(x(t))$, there is a sequence $\left\{\bar{T}_{n}\right\}$ tending to infinity with $d\left(x\left(\bar{T}_{n}\right), E\right) \rightarrow 0$ as $n \rightarrow \infty$. It is then possible to find sequences $\left\{t_{n}\right\}$ and $\left\{T_{n}\right\}$ tending to infinity with $d\left(x\left(t_{n}\right), E\right)=\varepsilon, d\left(x\left(T_{n}\right), E\right)=\eta / 2$, and $\eta / 2<$ $d(x(t), E) \leqslant \varepsilon$ if $T_{n} \leqslant t \leqslant t_{n}$.

If we compute the derivative of $H(t, x)$ along solutions of (1) we find (using (iii) in the definition of the pseudo-Liapunov function)

$$
H_{(1)}^{\prime}(t, x)=\operatorname{grad} H \cdot \mathcal{F}+\partial H / \partial t \text {. }
$$

In particular, if $T_{n} \leqslant t \leqslant t_{n}$, then $H_{(1)}^{\prime}(t, x) \leqslant P$. Integrating $H_{(1)}^{\prime}(t, x)$ from $T_{n}$ to $t_{n}$ we obtain

$$
H\left(t_{n}, x\left(t_{n}\right)\right)-H\left(T_{n}, x\left(T_{n}\right)\right) \leqslant P\left(t_{n}-T_{n}\right) .
$$

As $H\left(t_{n}, x\left(t_{n}\right)\right)>\lambda$ and $H\left(T_{n}, x\left(T_{n}\right)\right)<\lambda / 2$ we have

$$
\lambda-\lambda / 2<H\left(t_{n}, x\left(t_{n}\right)\right)-H\left(T_{n}, x\left(T_{n}\right)\right)<P\left(t_{n}-T_{n}\right)
$$

or

$$
t_{n}-T_{n} \geqslant \lambda / 2 P \stackrel{\text { def }}{=} T
$$

As $\mathcal{V}_{(1)}^{\prime}(t, x(\cdot)) \leqslant-L(x(t))$, for $t>t_{n}$ we have

$$
\begin{aligned}
\mathcal{V}(t, x(\cdot)) & \leqslant \mathcal{V}\left(t_{0}, \phi\right)-\int_{t_{0}}^{t} L(x(s)) d s \\
& \leqslant \mathcal{V}\left(t_{0}, \phi\right)-\sum_{i=1}^{n} \int_{T_{i}}^{t_{i}} L(x(s)) d s \\
& \leqslant \mathcal{V}\left(t_{0}, \phi\right)-\sum_{i=1}^{n} \int_{T_{i}}^{t_{i}} L_{0} d s \leqslant \mathcal{V}\left(t_{0}, \phi\right)-n L_{0} T
\end{aligned}
$$

for some $L_{0}>0$ by definition of $L$ being positive definite relative to $E$. Here, $\phi$ is the initial function for $x$. As $n \rightarrow \infty$, we contradict $\mathscr{V}>0$. This completes the proof. 
EXAMPLE 3. In Example 2, we can take $r(t)=0, V\left(t, x_{1}, x_{2}\right)=x_{2}^{2}+2 G\left(x_{1}\right)$ where

$$
G\left(x_{1}\right)=\int_{0}^{x_{1}} g(s) d s, \quad V_{(10)}^{\prime}=-2 \psi\left(t, x_{1}, x_{2}\right) x_{2}^{2},
$$

$H(t, x)=x_{2}^{2}$, and $H_{(10)}^{\prime}(t, x)=-2 \psi\left(t, x_{1}, x_{2}\right) x_{2}^{2}-2 x_{2} g\left(x_{1}\right)$. If for each $M$ $>0$, there exists $\alpha>0$ with $\psi\left(t, x_{1}, x_{2}\right) \geqslant \alpha$ when $\left|x_{2}\right| \leqslant M$ then the conditions of the theorem are satisfied and all bounded solutions approach the $x_{1}$-axis. If $G\left(x_{1}\right) \rightarrow \infty$ as $\left|x_{1}\right| \rightarrow \infty$, then all solutions are bounded. If $x_{1} g\left(x_{1}\right)$ $>0$ for $x_{1} \neq 0$, then an argument as in [2] yields all solutions bounded.

EXAMPLE 4. In Example 2, let $r(t)>0, r^{\prime}(t) \leqslant \alpha<b / 2,\left|g^{\prime}(x)\right| \leqslant L$, and $\left(x_{1}, x_{2}\right)=(x, y)$. Suppose $2 \psi(t, x, y) / r(t) \geqslant b+a / r(t)$ for some positive constants $a$ and $b$ with $4 L^{2} \leqslant b^{2}-2 \alpha b$.

Write the system as

$$
\begin{gathered}
x^{\prime}(t)=y(t), \\
y^{\prime}(t)=-\psi(t, x(t), y(t)) y(t)-g(x(t))+\int_{-r(t)}^{0} g^{\prime}(x(t+s)) y(t+s) d s
\end{gathered}
$$

and take

$$
\mathcal{V}(t, x(\cdot), y(\cdot))=2 G(x(t))+y^{2}(t)+\frac{b}{2} \int_{-r(t)}^{0}\left(\int_{s}^{0} y^{2}(t+u) d u\right) d s
$$

with $G(x)=\int_{0}^{x} g(s) d s$. We obtain

$$
\begin{aligned}
\mathcal{V}_{(11)}^{\prime}= & -2 \psi(t, x, y) y^{2}+2 y \int_{-r(t)}^{0} g^{\prime}(x(t+s)) y(t+s) d s \\
& +\frac{b}{2}\left[\int_{-r(t)}^{0}\left\{y^{2}(t)-y^{2}(t+s)\right\} d s+r^{\prime}(t) \int_{-r(t)}^{0} y^{2}(t+s) d s\right] .
\end{aligned}
$$

If we use the assumption on $\psi$ we find $\mathcal{V}_{(11)}^{\prime} \leqslant-a y^{2}(t)$.

Then $L(x, y)=-a y^{2}$ is negative definite relative to $E=\{(x, y) \mid y=0\}$. Here, $U=R \times R$.

Example 2 showed that $H=y^{2}$ is a pseudo-Liapunov function. By our result we conclude that all bounded solutions approach the $x$-axis. If $G(x) \rightarrow$ $\infty$ as $|x| \rightarrow \infty$, all solutions are bounded.

Theorem 2 is related to a result of Hale (cf. [7, pp. 118-126]) for autonomous functional differential equations $x^{\prime}(t)=\Re\left(x_{t}\right)$. In that reference, Hale and Somolinos apply the result to our system (11) when $r(t)$ is constant, $\psi(t, x, y)=a / r$ and $g(x)=(b / r) \sin x$. They conclude that bounded solutions approach invariant sets located on the $x$-axis. Hale's result was the natural generalization of the autonomous form of Theorem 0 to autonomous functional differential equations with finite lag. 
Corollary. Let $\mathfrak{V}$ be a functional with $\mathcal{V}_{(1)}^{\prime}(t, x) \leqslant-W_{4}(|x(t)|)$. Suppose there is a differentiable function $H:[0, \infty) \times R^{n} \rightarrow[0, \infty)$ with

$$
W_{2}(|x|) \leqslant H(t, x) \leqslant W_{3}(|x|)
$$

and $\operatorname{grad} H \cdot \mathcal{F}+\partial H / \partial t$ bounded above for any continuous function $x(t)$ which is defined and bounded on $[0, \infty)$. Then every bounded solution of (1) tends to zero as $t \rightarrow \infty$. If, in addition, $W_{1}(|x(t)|) \leqslant \mathcal{V}(t, x)$ and $\mathscr{V}(t, 0)=0$, then $x=0$ is asymptotically stable.

Proof. $H$ is a pseudo-Liapunov function when $E=\{0\}$ and $U=R^{n}$. Thus, by Theorem 2, all bounded solutions approach zero. With the additional hypotheses, the zero solution is Liapunov stable (Driver [4, p. 415]). As bounded solutions tend to zero we have asymptotic stability.

Example 0 is also an example of this corollary. The corollary complements Theorem 1 by allowing $H=H(t, x)$, but restricts the sign of $H$.

REMARK. If the conditions of Theorem 0 hold for (4), then the conditions of Theorem 2 also hold. To see this, let the conditions of Theorem 0 hold and let $\rho$ be the distance function from a point $x \in R^{n}$ to $E$. In [9] Langenhop points out that $|\rho(x)-\rho(y)| \leqslant|x-y|$, a global Lipschitz condition, while if $E$ is convex, then $\rho^{2}$ is actually differentiable. Let $H(t, x)=\rho(x)$ and compute $\rho_{(4)}^{\prime}$. We have

$$
\begin{aligned}
& \mid \varlimsup_{h \rightarrow 0^{+}} \frac{1}{h}\{\rho(x+h P(t, x))-\rho(x)\} \mid \\
& \leqslant \varlimsup_{h \rightarrow 0^{+}} \frac{1}{h}|x+h P(t, x)-x|=|P(t, x)|
\end{aligned}
$$

which is bounded for $x$ bounded. Thus, we easily see that $\rho$ is a pseudoLiapunov function for $E$ and (4).

We turn now to the system

$$
x^{\prime}=\mathcal{G}\left(t, x_{t}\right)
$$

and obtain a generalization of the classical result of Yoshizawa [12, p. 191]. For completeness we supply details of the notation of Yoshizawa [12, pp. 181-191].

For a given $h>0, C$ denotes the space of continuous functions mapping the interval $[-h, 0]$ into $R^{n}$. If $\phi \in C$, then $\|\phi\|=\sup _{-h<\theta<0}|\phi(\theta)| . C_{M}$ denotes the set of $\phi \in C$ with $\|\phi\|<M$. If $x$ is a continuous function, $x$ : $[-h, A) \rightarrow R^{n}$ for $A>0$, and if $t$ is a fixed number in $[0, A)$, then $x_{t}$ is the (shifted) restriction of $x(u)$ to the interval $[t-h, t]$; thus $x_{t}(\theta)=x(t+\theta)$ for $-h<\theta<0$, shifted back to the interval [ $-h, 0]$.

We ask that $\mathcal{G}:[0, \infty) \times C_{M} \rightarrow R^{n}$ be continuous and take bounded sets into bounded sets.

It has become standard to ask that $\mathcal{V}_{(3)}^{\prime}(t, x)<-W_{4}(|x(t)|)$. It should be 
clear in the following proof that a similar result and proof hold if $\mathcal{V}_{(3)}^{\prime}(t, x)<$ - $W_{4}\left(\left\|x_{t}\right\|_{\left(h_{1}, h_{2}\right)}\right)$ where

$$
\left\|x_{t}\right\|_{\left(h_{1}, h_{2}\right)}=\sup _{-h_{1}<\theta<-h_{2}}\left|x_{t}(\theta)\right|
$$

and $-h<-h_{1}<-h_{2}<0$. The latter was Yoshizawa's form.

TheOREM 3. Let $\mathfrak{V}:[0, \infty) \times C_{M} \rightarrow[0, \infty)$ be continuous, satisfy a Lipschitz condition in the second argument, and let

$$
W_{1}(|x(t)|) \leqslant \mathcal{T}\left(t, x_{t}\right)<W_{2}\left(\left\|x_{t}\right\|\right)
$$

with $\mathcal{V}_{(3)}^{\prime}\left(t, x_{t}\right)<-W_{4}(|x(t)|)$. Let $E=\{0\}, U$ be a neighborhood of $E$ in $R^{n}$, $H:[0, \infty) \times U \rightarrow[0, \infty)$ with $W_{5}(|x|) \leqslant H(t, x) \leqslant W_{6}(|x|)$. If $H_{(3)}^{\prime}(t, x)$ is bounded above for $x \in U$ and $x_{t} \in C_{M}$, then $x=0$ is uniformly asymptotically stable for (3).

Remark. When $\mathcal{G}\left(t, x_{t}\right)$ is bounded for $x_{t} \in C_{M}$, then Yoshizawa's hypotheses for UAS are identical to those in our first sentence. Our result replaces $\mathcal{G}$ bounded by the existence of $H$ with $H^{\prime}$ bounded above. To see that Yoshizawa's result is a corollary of ours, if $\mathcal{G}$ is bounded for $x_{t} \in C_{M}$, take $H(t, x)=x_{1}^{2}+\cdots+x_{n}^{2}$ and obtain

$$
H_{(3)}^{\prime}(t, x)<\left|\mathcal{G}\left(t, x_{t}\right)\right|(2 n M)
$$

for $|x|<M$ and $x_{t} \in C_{M}$.

Proof of Theorem 3. Let $\varepsilon>0$ be given with $\varepsilon<M$. There exists $\delta>0$ with $W_{2}(\delta)<W_{1}(\varepsilon)$. If $t \geqslant t_{0}$ and if $\|\phi\|<\delta$, then for $x(t)=x\left(t, t_{0}, \phi\right)$ we have

$$
\begin{aligned}
W_{1}(|x(t)|) & \leqslant \mathcal{V}\left(t, x_{t}\right) \leqslant \mathcal{V}\left(t_{0}, \phi\right) \leqslant W_{2}(\|\phi\|) \\
& <W_{2}(\delta)<W_{1}(\varepsilon) \text { so }|x(t)|<\varepsilon .
\end{aligned}
$$

This shows uniform stability.

By the uniform stability, $\eta>0$ may be chosen so that $t_{0} \geqslant 0, t>t_{0}$, and $\|\phi\|<\eta$ implies $\left|x\left(t, t_{0}, \phi\right)\right|<M / 2$.

Now let $\varepsilon>0$ be given. To complete the proof we must find $T>0$ such that $t_{0}>0,\|\phi\|<\eta$, and $t>t_{0}+T$ imply $\left|x\left(t, t_{0}, \phi\right)\right|<\varepsilon$. For this $\varepsilon>0$, find $\delta>0$ by uniform stability so that $t_{0}>0,\|\phi\|<\delta$, and $t>t_{0}$ imply $\left|x\left(t, t_{0}, \phi\right)\right|<\varepsilon$.

Let $t_{0} \geqslant 0$ be arbitrary and consider intervals

$$
\begin{array}{r}
I_{1}=\left[t_{0}, t_{0}+h\right], \quad I_{2}=\left[t_{0}+h, t_{0}+2 h\right], \ldots, \\
I_{k+1}=\left[t_{0}+k h, t_{0}+(k+1) h\right], \ldots
\end{array}
$$

If $\|\phi\|<\eta$ and $x(t)=x\left(t, t_{0}, \phi\right)$, then for each $i$ either $|x(t)|<\delta$ on $I_{i}$ (and hence $|x(t)|<\varepsilon$ for $\left.t>t_{0}+i h\right)$ or there exists $t_{i} \in I_{i}$ with $\left|x\left(t_{i}\right)\right|>\delta$.

Now there exists $\gamma>0$ such that $W_{6}(\gamma)<W_{5}(\delta)$. Also, there exists $\bar{T}>0$ 
such that if $\left|x\left(t_{i}\right)\right| \geqslant \delta$ then $|x(t)| \geqslant \gamma$ for $t_{i}-\bar{T} \leqslant t<t_{i}$. To see this, if for some $\bar{t}_{i}<t_{i}$ we have $\left|x\left(\bar{t}_{i}\right)\right|=\gamma,\left|x\left(t_{i}\right)\right| \geqslant \delta$, and $\gamma \leqslant|x(t)|$ for $\bar{t}_{i}<t \leqslant t_{i}$, then we have $H_{(3)}^{\prime}(t, x(t))<P$ for some $P>0$ and so

$$
\begin{aligned}
W_{5}\left(\left|x\left(t_{i}\right)\right|\right) & \leqslant H\left(t_{i}, x\left(t_{i}\right)\right) \leqslant H\left(\bar{t}_{i}, x\left(\bar{t}_{i}\right)\right)+P\left(t_{i}-\bar{t}_{i}\right) \\
& \leqslant W_{6}\left(\left|x\left(\bar{t}_{i}\right)\right|\right)+P\left(t_{i}-\bar{t}_{i}\right)
\end{aligned}
$$

or

so that

$$
W_{5}(\delta) \leqslant W_{6}(\gamma)+P\left(t_{i}-\bar{t}_{i}\right)
$$

$$
\bar{T} \stackrel{\text { def }}{=}\left[W_{5}(\delta)-W_{6}(\gamma)\right] / P \leqslant t_{i}-\bar{t}_{i} \text {. }
$$

Let $Q=\min [\bar{T}, h]$, pick $N>W_{2}(\eta) / W_{4}(\gamma) Q$, and choose $T=2 N h$. We now show that $|x(t)|<\delta$ for some $t$ in $\left[t_{0}, t_{0}+T\right]$ so that $|x(t)|<\varepsilon$ for $t>t_{0}+T$. If such a $t$ does not exist, then in each $I_{i}$ there is a $t_{i}$ with $\left|x\left(t_{i}\right)\right|>\delta$ and, hence, $|x(t)| \geqslant \gamma$ for $t_{i}-Q<t<t_{i}$. Thus, $\mathfrak{V}_{(3)}^{\prime}\left(t, x_{t}\right)<$ $-W_{4}(\gamma)$ for $t_{i}-Q<t<t_{i}$. Let $t>t_{2 N}$ so that

$$
\begin{aligned}
\mathcal{V}\left(t, x_{t}\right) & \leqslant \mathcal{V}\left(t_{0}, \phi\right)-\sum_{i=1}^{N} \int_{t_{2 i}-Q}^{t_{2 i}} W_{4}(\gamma) d t \\
& =\mathcal{V}\left(t_{0}, \phi\right)-N Q W_{4}(\gamma) \leqslant W_{2}(\eta)-N Q W_{4}(\gamma)<0
\end{aligned}
$$

a contradiction. In our integration it was necessary to skip the intervals $\left[t_{i}-Q, t_{i}\right]$ with $i$ odd to avoid possible overlap.

EXAMPLE 5. Consider the scalar equation $x^{\prime}(t)=-a(t) x(t)+b(t) x(t-$ $r(t))$ with $a(t)>a_{0}>0, r(t) \geqslant 0, r^{\prime}(t) \leqslant \alpha<1, r(t)<h$ for some $h>0$, and $|b(t)|<a_{1} \sqrt{1-\alpha}<a_{0} \sqrt{1-\alpha}$ for some $a_{1}>0$.

Take

$$
\mathscr{V}(t, x(\cdot))=x^{2}(t)+a_{0} \int_{t-r(t)}^{t} x^{2}(s) d s
$$

so that

$$
\begin{aligned}
\mathcal{V}^{\prime}= & -2 a(t) x^{2}(t)+2 b(t) x(t) x(t-r(t)) \\
& +a_{0}\left[x^{2}(t)-x^{2}(t-r(t))\left(1-r^{\prime}(t)\right)\right] \\
< & {\left[-2 a(t)+a_{0}\right] x^{2}(t)+2|b(t)||x(t) x(t-r(t))| } \\
& +a_{0}(\alpha-1) x^{2}(t-r(t)) \\
< & -a_{0} x^{2}(t)+2|b(t)||x(t) x(t-r(t))|+a_{0}(\alpha-1) x^{2}(t-r(t)) \\
< & -a_{0}\left[1-\left\{b^{2}(t) / a_{0}^{2}(1-\alpha)\right\}\right] x^{2}(t),
\end{aligned}
$$

as may be seen by completing the square. This yields $\mathcal{V}^{\prime}(t, x) \leqslant-\gamma x^{2}$ for some $\gamma>0$. We take $H=x^{2}$ and have $H^{\prime} \leqslant 2 a_{1} \sqrt{1-\alpha}|x(t) x(t-r(t))|$. The conditions of Theorem 3 are satisfied and $x=0$ is UAS. 
REMARK. In [3] we showed that $\mathcal{G}$ need not be bounded in Yoshizawa's result provided that

$$
\mathcal{V}(t, x) \leqslant W_{7}(|x(t)|)+W_{8}\left(||\left|x_{t}\right| \mid\right)
$$

where $\left\|\left|x_{t} \|\right|\right.$ is the $L^{2}$-norm. If, for example,

$$
\checkmark(t, x)=\sup _{-h<\theta<0}|x(t+\theta)|
$$

then an $L^{2}$ norm could not be used. Converse theorems yield the existence of Liapunov functionals requiring the sup-norm (cf. [8, p. 56]).

REMARK. In arguments of the type given above it is well known that the requirement that the $W_{i}$ be strictly increasing is entirely unnecessary. However, without that condition the arguments do become rather protracted.

Acknowledgments. The author is indebted to Professor C. E. Langenhop for pointing out [9] which allowed us to show that Theorem 0 is a consequence of Theorem 2 . He also read the manuscript and made a number of helpful suggestions.

\section{REFERENCES}

1. H. A. Antosiewicz, A survey of Lyapunov's second method, Ann. of Math. Studies, no. 41, Contributions to the Theory of Nonlinear Oscillations, vol. 4, Princeton Univ. Press, Princeton, N. J., 1958, pp. 141-166.

2. T. A. Burton, The generalized Lienard equation, SIAM J. Control 3 (1965), 223-230.

3. , Uniform asymptotic stability for functional differential equations, Proc. Amer. Math. Soc. 68 (1978), 195-199.

4. Rodney D. Driver, Existence and stability of solutions of a delay-differential system, Arch. Rational Mech. Anal. 10 (1962), 401-426.

5. Existence theory for a delay-differential system, Contributions to Differential Equations 1 (1963), 317-336.

6. John R. Haddock, On Liapunov functions for nonautonomous systems, J. Math. Anal. Appl. 47 (1974), 599-603.

7. Jack Hale, Theory of functional differential equations, Springer, New York, 1977.

8. V. Lakshmikantham and S. Leela, Differential and integral inequalities. Vol. II in Mathematics in Science and Engineering, Vol. 55, Academic Press, New York, 1969.

9. C. E. Langenhop, Differentiability of the distance to a set in Hilbert space, J. Math. Anal. Appl. 44 (1973), 620-624.

10. J. P. La Salle, Stability theory for ordinary differential equations, J. Differential Equations 4 (1968), 57-65.

11. Stability of nonautonomous systems, Nonlinear Analysis, Theory, Methods and Appl. 1 (1976), 83-91.

12. T. Yoshizawa, Stability by Liapunov's second method, Math. Soc. Japan, Tokyo, 1966.

13. Asymptotic behavior of solutions of a system of differential equations, Contributions to Differential Equations 1 (1963), 371-387.

Department of Mathematics, Southern Illinois University, Carbondale, Illinois 62901 